\title{
Avaliação ginecológica e citológica em vacas Holandesas com mais de três repetições de cio
}

\section{Gynecological and cytological evaluation in Holstein cows with more than three repetitions of estrus}

\author{
Cícero Fleury Guedes Martins ${ }^{1}$; Patricia Magalhães de Oliveira ${ }^{2 *}$; \\ Nayara Resende Nasciutti²; Vinícius de Morais Barbosa ${ }^{3}$; \\ Mariana Borges Ferreira'; Milton Maturana Filho ${ }^{4}$; Ricarda Maria dos Santos ${ }^{5}$; \\ Francisco Cláudio Dantas Mota ${ }^{5}$ João Paulo Elsen Saut ${ }^{5}$
}

\section{Resumo}

A eficiência reprodutiva é um dos principais fatores envolvidos na viabilidade econômica do setor de criação de bovinos. Objetivou-se realizar a avaliação ginecológica e citológica em vacas Holandesas com mais de três repetições de cio. O experimento foi desenvolvido em seis propriedades leiteiras comerciais, com manejos sanitários e reprodutivos semelhantes e assistidas por médicos veterinários. Foram incluídas apenas vacas da raça Holandesa, até cinco parições e mais de três repetições de cio consecutivas. Na propriedade, os animais foram identificados e procedidos o exame ginecológico e citologia endometrial. No presente estudo foram avaliadas 77 vacas e as causas reprodutivas encontradas em vacas Holandesas sujeitas ao descarte involuntário foram: endometrite clínica com secreção vaginal purulenta e mais que 3\% de neutrófilos (7,79\%), endometrite clínica com secreção vaginal purulenta e menos que 3\% de neutrófilos $(2,6 \%)$, endometrite subclínica (10,4\%), urovagina $(1,3 \%)$ e $77,9 \%$ que não se sabe a causa, porém não está associado aos distúrbios reprodutivos avaliados. Concluiu-se que: a) o índice de vacas que não apresentaram a causa diagnosticada é muito alto e isto interfere diretamente na eficiência reprodutiva; b) o exame proposto diagnosticou $22,1 \%$ de distúrbios clínicos que poderiam ser evitados ou tratados e desta forma reduzindo o descarte involuntário.

Palavras-chave: Bovinos, endometrite clínica, endometrite subclínica, urovagina

\footnotetext{
Abstract

The objective of this study was to evaluate the gynecological examination and endometrial cytology in Holstein cows with more than three repetitions of estrus. The experiment was conducted in six commercial dairy herds both with similar reproductive and sanitary managements and assisted by veterinarians. It was included only Holstein cows up to five calving and more than three consecutive repetitions of estrus. Seventy-seven cows were identified and carried gynecological examination and endometrial cytology. The reproductive causes found in this study, subject to involuntary culling, were: clinical endometritis

${ }^{1}$ Médicos Veterinários autônomos, Uberlândia, MG. E-mail: cirsuf@gmail.com; maribferreira@yahoo.com.br

${ }^{2}$ Discentes do Programa de Pós-graduação em Ciências Veterinárias, Universidade Federal de Uberlândia, UFU, Uberlândia, MG. E-mail: pmo.veterinaria@yahoo.com.br; nayaranasciutti@yahoo.com.br

${ }^{3}$ Discente do Curso de Medicina Veterinária da Faculdade de Medicina Veterinária, FAMEV/UFU, Uberlândia, MG. E-mail: vinicius.barbosa6@hotmail.com

${ }^{4}$ Discente do Programa de Pós-graduação em Reprodução Animal, Universidade de São Paulo, USP, Pirassununga, SP. E-mail: milton.maturana@gmail.com

${ }^{5}$ Profs. da FAMEV/UFU, Uberlândia, MG. E-mail: ricasantos@famev.ufu.br; dantasmota@famev.ufu.br; jpsaut@famev.ufu.br

* Autor para correspondência
} 
with purulent vaginal discharge and more than 3\% neutrophils $(7.79 \%)$, clinical endometritis with purulent vaginal discharge and less than 3\% neutrophils (2.6\%), subclinical endometritis $(10.4 \%)$, urovagina $(1.3 \%)$ and $77.9 \%$ cows with unknown causes and they were not associated with reproductive disorders evaluated. It was concluded: a) the rate of cows without diagnosis is very high and it interferes directly to the reproductive performance; b) the proposed examination diagnosed $22.1 \%$ of clinical disorders that might be prevented or treated and thus decreasing the involuntary culling.

Key words: Cattle, clinical endometritis, subclinical endometritis, urovagina

\section{Introdução}

A eficiência reprodutiva é um dos principais fatores envolvidos na viabilidade econômica do setor de criação de bovinos. Problemas na reprodução têm significativo impacto na produção leiteira, número de bezerros nascidos por ano, intervalo entre gerações e, consequentemente, na rentabilidade da fazenda (GROOMS, 2010).

$\mathrm{O}$ risco de uma vaca não emprenhar após um período de serviço ideal pode ser atribuído a distúrbios clínicos detectáveis como as infecções uterinas (metrite e endometrite clínica) e distúrbios não detectáveis. Caso persistam por três inseminações consecutivas sem sinais clínicos aparentes, são ora denominadas vacas repetidoras de cio. A incidência dessa síndrome varia de acordo com a região, ambiente e manejo dos rebanhos e tem sido relatado a incidência de $14 \%$ e $62 \%$ em vacas Holandesas (YUSUF et al., 2010, 2012), 20,99\% (87.823/418.383) em rebanhos com predominância de vacas Holandesas (BONNEVILLE-HÉBERT et al., 2011) e $10,1 \%$ de 57.616 vacas em rebanhos leiteiros na Suécia (GUSTAFSSON; HEMANUELSSON, 2002).

Espera-se que o animal retorne a sua atividade cíclica ovariana rapidamente após o parto, além da ocorrência das etapas de involução uterina, regeneração do endométrio e eliminação da infecção bacteriana, sendo o estímulo inicial para ocorrer estas mudanças à expulsão do feto, das membranas fetais e da lóquia (SHELDON et al., 2008). Caso haja deficiência em uma ou mais etapas deste processo e a presença de bactérias patogênicas, poderá então haver a instalação de infecções uterinas e, possivelmente, a infertilidade do animal
(SHELDON; DOBSON, 2004).

A endometrite clínica atinge cerca de cinco a $15 \%$ de vacas leiteiras no pós-parto e é caracterizada por inflamação do endométrio a partir dos 20 dias pós-parto (dpp) com a presença de descarga vaginal purulenta ou aos 26 dpp com descarga vaginal mucopurulenta, sem a presença de sinais sistêmicos. A endometrite pode persistir se não tratada adequadamente e está associada com o atraso na involução uterina, falhas na concepção na primeira inseminação, vacas repetidoras de cio e descartes involuntários (SHELDON et al., 2008; DUBUC et al., 2010; BONNEVILLE-HÉBERT et al., 2011).

Problemas reprodutivos e falhas na concepção tem sido a causa primária no descarte involuntário de animais nas propriedades leiteiras, e isto reduz a lucratividade do rebanho, pois não está associada à produção leiteira. Vacas repetidoras de cio apresentam taxas de descarte de $35 \%$ em relação a $8 \%$ das vacas sem alterações clínicas e reprodutivas no pós-parto (OPSOMER et al., 2000; BONNEVILLE-HÉBERT et al., 2011). Diante destes fatos, objetivou-se realizar a avaliação ginecológica e citológica em vacas Holandesas com mais de três repetições de cio consecutivas após o período voluntário de espera.

\section{Material e Métodos}

O experimento foi desenvolvido, entre novembro de 2011 e abril de 2012, em seis propriedades leiteiras comerciais, sendo uma em Uberlândia, Minas Gerais, e cinco em Pirassununga, São Paulo, com manejos sanitários e reprodutivos semelhantes e assistidas 
por médicos veterinários. Foram incluídas apenas vacas da raça Holandesa, até cinco parições, três repetições de cio consecutivas após as respectivas inseminações artificiais (I.A.) e entre 100 a 200 dias pós-parto (dpp). Além disso, que estas vacas não apresentassem no seu histórico reprodutivo distocia, retenção de placenta ou metrite puerperal aguda e no histórico clínico cetose, hipocalcemia, deslocamento de abomaso, timpanismo, pneumonia, afeccções podais ou quaisquer outras doenças que tivessem que ter sido medicadas. Vacas com tratamento de antibioticoterapia local para mastite foram incluídas. Na propriedade, no dia da visita, os animais com o histórico reprodutivo e clínico compatível eram submetidos à avaliação do escore de condição corporal (ECC), exame ginecológico e citologia endometrial. Classificou-se o ECC de 1 a 5 (FERREIRA, 1990) e a média dos animais avaliados foi de $3,1 \pm 0,8(1,75-5,00)$.

As detecções de cio foram feitas por observação visual, pelo menos duas vezes ao dia, no período de 60 minutos cada avaliação, e em apenas uma propriedade com auxílio do sistema pedômetro. As I.A. foram realizadas por inseminadores experientes e o sêmen utilizado era armazenado em botijões criogênicos contendo nitrogênio líquido, e proveniente de empresas registradas e habilitadas pelo Ministério da Agricultura.

No exame ginecológico realizou-se a inspeção direta de vulva e vestíbulo vulvar, palpação retal, ultrassonografia, vaginoscopia e coleta de secreção vaginal. Avaliou-se a vulva conforme sua posição em relação ao púbis (VASCONCELOS et al., 2000) e angulação determinada pela inspeção dos lábios vulvares (GAUTAM; NAKAO, 2009).

No exame de palpação e ultrassonografia retal, com transdutor retal linear de 5-10 $\mathrm{MHz}$ (DP-2200VET®, Mindray, Shenzhen, China), observaram-se: localização uterina, presença de líquido no lúmen uterino, simetria dos cornos e estruturas no ovário. Após, procedeu-se a vaginoscopia, com auxílio de espéculo tubular específico para bovinos (Walmur ${ }^{\circledR}$ ), para o exame da vagina e óstio cervical externo em relação à presença de lesão e abertura do óstio cervical.

Por último, colheu-se e inspecionou-se a característica da secreção vaginal, com prévia limpeza da vulva com papel toalha, em seguida, utilizando luva de palpação nova, introduziase a mão na vagina, para remover o conteúdo. A classificação da secreção foi adaptada de Sheldon et al. (2008): a) secreção limpa e translúcida; b) secreção com flocos de pus ou esbranquiçada; c) secreção contendo $<50 \%$ de pus; d) secreção contendo $>50 \%$ de pus.

Definiu-se urovagina quando a secreção colhida apresentava urina ou um misto de urina e muco. Avaliou-se, com auxílio da vaginoscopia, a extensão de cobertura do óstio externo da cérvix pela urina ou misto de urina/muco em: a) grau leve: pequena quantidade apenas no assoalho da vagina, mas que não atingia o óstio externo da cérvix; $b$ ) grau moderado: quantidade apreciável cobrindo até a metade do óstio externo da cérvix; c) grau severo: grande quantidade cobrindo pelo menos metade ou totalmente o óstio externo da cérvix (GAUTAM; NAKAO, 2009).

$\mathrm{Na}$ citologia endometrial as amostras foram colhidas pela técnica de escova endometrial (cytobrush), de acordo com Kaufmann et al. (2009). Após corou-se pelo método de May-Grunwald Giemsa para avaliação microscópica. A análise da citologia endometrial foi feita pela avaliação do percentual de neutrófilos, a partir da contagem de 200 células, na magnitude de 1000x, sendo a leitura feita por dois observadores e classificando como endometrite subclínica a presença acima de 3\% de neutrófilos (SALASEL; MOKHTARI; TAKTAZ, 2010).

Para a análise estatística, utilizou-se o programa Minitab 15 (Minitab Inc, Pennsylvania, USA) e os dados foram apresentados em porcentagem, média aritmética e desvio-padrão. Para verificar se havia diferença na frequência de ECC e na presença de 
corpo lúteo entre os grupos: endometrite clínica, endometrite subclínica e sem alteração clínica utilizou-se o teste de qui-quadrado $(\mathrm{p}<0,05)$; para comparar a mediana dos ECC realizou-se o teste de Kruskal-Wallis (Anova não-paramétrica) $(\mathrm{p}<0,05)$ (VIEIRA, 2003).

\section{Resultados e Discussão}

Foram avaliadas 77 vacas com histórico de mais de três inseminações consecutivas, das quais nenhuma apresentou problemas relacionados com vulva, vestíbulo vulvar, vagina e óstio cervical externo. Apenas uma vaca foi diagnosticada com urovagina de grau moderado. A urovagina predispõe à entrada de urina no útero causando infertilidade devido à ação espermicida, além de causar vaginite, cervicite e endometrite crônica (GILBERT et al., 1989).

Das vacas incluídas no experimento, 10,4\% (8/77) apresentaram endometrite clínica (Tabela 1). A ocorrência desta enfermidade não seria esperada nestas vacas com mais de $100 \mathrm{dpp}$, e isto pode ser decorrente mais comumente da contaminação uterina no momento da inseminação artificial, falta de diagnóstico das infecções uterinas, falha do tratamento proposto e resistência dos microrganismos envolvidos nas infecções uterinas aos antibióticos (SANTOS et al., 2010; SALASEL; MOKHTARI; TAKTAZ, 2010).

Tabela 1. Incidência de distúrbios encontrados em vacas Holandesas com mais de três inseminações artificiais consecutivas.

\begin{tabular}{ccc}
\hline DISTÚRBIOS ENCONTRADOS & $\mathbf{N}^{\mathbf{0}}$ & $\mathbf{\%}$ \\
\hline Endometrite Clínica (Secreção $+;>3 \%$ neutrófilos) & $6 / 77$ & $7,79 \%$ \\
Endometrite Clínica (secreção $+;<3 \%$ neutrófilos) & $2 / 77$ & $2,60 \%$ \\
Endometrite Subclínica & $8 / 77$ & $10,4 \%$ \\
Urovagina & $1 / 77$ & $1,30 \%$ \\
Sem alteração* & $60 / 77$ & $77,9 \%$ \\
\hline
\end{tabular}

Nota: Secreção + (presença de secreção vaginal mucopurulenta ou purulenta); $<3 \%$ ou $>3 \%$ de neutrófilos (exame de citologia endometrial - cytobrush); * Sem alteração no exame clínico proposto na pesquisa.

Fonte: Elaboração dos autores.

A avaliação clínica da involução uterina foi realizada pelos veterinários particulares das propriedades e vacas com infecções uterinas e tratadas não participaram do experimento. No entanto, não se verificou consenso nos critérios de definição e classificação de endometrite clínica entre as propriedades. De acordo com Gilbert et al. (2005), não há um consenso na definição de endometrite na literatura veterinária e Paisley, Mickelsen e Anderson (1986) acreditam que há ignorância quanto ao conhecimento das secreções uterinas pós-parto e isto induz aos erros observados no diagnóstico.

Do total de vacas com secreção vaginal mucopurulenta ou purulenta, 75\% (6/8) apresentaram exame de citologia endometrial compatível com endometrite clínica, isto é, presença de neutrófilos acima de 3\% (Tabela 1). No entanto, 25\% (2/8) apresentaram citologia endometrial com menos de $3 \%$ de neutrófilos o que sugere que estes animais apresentem vaginite ou cervicite (DEGUILLAUME et al., 2012) e justificaria a presença desta secreção no interior da vagina.

De acordo com Dubuc et al. (2010), a terminologia endometrite clínica pode não ser a mais indicada, optando-se pela denominação de Descarga Vaginal Purulenta (PVD), pois verificaram que vacas diagnosticadas com endometrite clínica, 
através da secreção vaginal, aos 35 e 56 dpp, apresentavam somente 38 e $36 \%$ do exame de citologia endometrial compatível, respectivamente. Conforme Deguillaume et al. (2012) a presença (ausência) de cervicite não é indicativa da presença (ausência) de endometrite. Ressaltam que a inflamação endocervical afeta a concepção e que a avaliação geral do trato genital apresenta maior benefício ao desempenho reprodutivo do que apenas a inflamação endometrial.

A porcentagem de neutrófilos no útero diminui fisiologicamente com o decorrer do pós-parto (SENOSY et al., 2012). No presente estudo, em animais com mais de $100 \mathrm{dpp}$, seria esperado a presença de secreção vaginal limpa e clara e com menos de 3\% de neutrófilos, porém foram encontradas $10,4 \%(8 / 77)$ das vacas com muco vaginal claro e com mais de 3\% de neutrófilos, sendo diagnosticadas com endometrite subclínica.

Afora a possibilidade de um diagnóstico não eficiente de endometrite clínica e a persistência desta infecção uterina no decorrer do pós-parto, outros fatores descritos como predisponentes para a endometrite subclínica como distocia, partos gemelares, abortos, retenção dos anexos fetais e outras infecções uterinas (SALASEL; MOKHTARI; TAKTAZ, 2010) podem ser descartados no presente experimento. A exclusão destes fatores justifica um menor índice de endometrite subclínica observado $(10,4 \%)$ quando comparado à prevalência encontrada de $52,7 \%$ em vacas repetidoras de cio por Salasel, Mokhtari e Taktaz (2010).

Ao considerar, no presente trabalho, apenas vacas repetidoras de cio de acordo com a definição de Salasel, Mokhtari e Taktaz (2010), ou seja, a somatória dos grupos com endometrite subclínica e dos que não apresentaram nenhuma alteração clinica no exame proposto (Tabela 1), sendo o total de 68 animais, a incidência de endometrite subclínica aumentou para 11,8\% (8/68), mesmo assim permanecendo bem menor ao descrito pelos pesquisadores (SALASEL; MOKHTARI; TAKTAZ, 2010).
Neste estudo, 77,9\% (60/77) (Tabela 1) das vacas não apresentaram nenhum distúrbio aparente e citologia endometrial com neutrófilos inferior a $3 \%$. A repetição de cio após o $3^{\circ}$ serviço não apresenta causa única e pode estar relacionada com o manejo da propriedade, nutrição e conforto animal. O estresse de qualquer natureza pode prejudicar o transporte dos espermatozóides no útero e na tuba, bem como provocar a morte do embrião. A inseminação realizada em horário inadequado proporciona o encontro de ovócito e/ ou espermatozóides envelhecidos, imaturos ou degenerados (FERREIRA, 2010).

A média geral de ECC nos animais foi de 3,08 $\pm 0,75$, e não houve diferença na média $(p=0,761)$ entre os animais com endometrite clínica $(2,94 \pm$ $0,75)$, endometrite subclínica $(2,94 \pm 0,82)$ e sem alteração clínica $(3,12 \pm 0,75)$ e nem diferença na frequência de ECC $(p=0,9424)$ dentro dos mesmos grupos (Tab. 2).

Os valores sugeridos de ECC para vacas da raça Holandesa são 3,5 (3,25-3,75), 3,0 (2,5-3,25), 3,25 $(2,75-3,25)$ e $3,5(3,0-3,5)$, respectivamente, no parto e início, meio e fim da lactação (FERGUSON; GALLIGAN; THOMSEN, 1994). A média das vacas no presente experimento, consideradas no meio da lactação, estão dentro dos valores esperados. Entretanto, ao se avaliar por extratos (Tab. 2), verifica-se que $37,5 \%$ das vacas com endometrite clínica e endometrite subclínica e 30\% das vacas sem alteração clínica estavam abaixo do ECC 2,5.

O ECC tem alta relação com o desempenho reprodutivo, vacas magras apresentam maiores problemas reprodutivos, de modo que aumentando o ECC há correlação positiva com intervalo de parto ao primeiro cio e primeiro serviço, taxa de prenhez à primeira cobertura e correlação negativa com número de serviços por vaca (PRYCE; COFFEY; BROTHERSTONE, 2000). De acordo com Ferguson, Galligan e Thomsen (1994), independente das vacas serem muito gordas ou magras, elas correm o risco de ter problemas metabólicos e 
doenças no pós-parto, redução na produção leiteira e taxa de concepção.

Ao se comparar os resultados com Corassin et al. (2009) que trabalharam com vacas Holandesas na mesma região e com sistema de produção e manejo nutricional semelhantes, os valores médios do ECC foram inferiores em vacas no primeiro serviço até os 150 dias $(3,28 \pm 0,53)$, considerados dentro da normalidade, além das vacas estarem bem mais adiantadas nos dias de lactação e já apresentarem ECC mais elevado.
Neste experimento verificou-se a presença de corpo lúteo em aproximadamente $70 \%$ das vacas (Tabela 2), o que demonstra que estas vacas estavam ciclando no dia da avaliação. Várias causas podem desencadear a repetição de cio em vacas, como distúrbios reprodutivos (endometrite clínica, cervicite, vaginite, endometrite subclínica e urovagina), ou mesmo falhas de manejo da propriedade como a não identificação correta do cio, logo, cuidados no pré e pós-parto tornam-se necessários a fim de se evitar perdas econômicas e aumentar a produtividade.

Tabela 2. Avaliação do escore de condição corporal (ECC) e presença de corpo lúteo em vacas Holandesas com mais de três inseminações artificiais consecutivas que apresentaram endometrite clínica, endometrite subclínica ou sem alterações clínicas.

\begin{tabular}{ccccc}
\hline & & $\begin{array}{c}\text { Endometrite } \\
\text { clínica }\end{array}$ & $\begin{array}{c}\text { Endometrite } \\
\text { subclínica }\end{array}$ & $\begin{array}{c}\text { Sem alteração } \\
\text { clínica* }\end{array}$ \\
\cline { 3 - 5 } & $\leq 2,5$ & $37.50 \%(3 / 8)^{\mathrm{a}}$ & $37.50 \%(3 / 8)^{\mathrm{a}}$ & $30 \%(18 / 60)^{\mathrm{a}}$ \\
$\mathrm{ECC}$ & $2,5<\mathrm{X} \leq 3,75$ & $50 \%(4 / 8)$ & $50 \%(4 / 8)$ & $48.33 \%(29 / 60)$ \\
& $\geq 4,0$ & $12.50 \%(1 / 8)$ & $12.50 \%(1 / 8)$ & $21.67 \%(13 / 60)$ \\
Presença de corpo & & & & \\
lúteo & $\mathrm{Sim}$ & $87.50 \%(7 / 8)^{\mathrm{a}}$ & $75 \%(6 / 8)^{\mathrm{a}}$ & $71.67 \%(43 / 60)^{\mathrm{a}}$ \\
& $\mathrm{Não}$ & $12.50 \%(1 / 8)$ & $25 \%(2 / 8)$ & $28.33 \%(17 / 60)$ \\
\hline
\end{tabular}

Nota: * Sem alteração clínica no exame clínico proposto na pesquisa.

Fonte: Elaboração dos autores.

Concluiu-se na avaliação ginecológica e citológica de vacas Holandesas com mais de três repetições de cio, em propriedades leiteiras comerciais, que: a) o índice de vacas que não apresentaram a causa diagnosticada é muito alto e interfere diretamente na eficiência reprodutiva; b) o exame proposto diagnosticou $22,1 \%$ de distúrbios clínicos que poderiam ser evitados ou tratados e desta forma reduzindo o descarte involuntário.

Este trabalho foi aprovado pelo comitê de bioética com o protocolo CEUA/UFU 143/11.

\section{Agradecimentos}

Os autores agradecem à Faculdade de Medicina
Veterinária da Universidade Federal de Uberlândia e FAPEMIG pelo auxílio financeiro; ao apoio da FZEA-USP e Fazenda da Aeronáutica em Pirassununga-SP por ceder o espaço e animais e, em especial, ao Doutor Ubiraem Mário Schalch por todo o apoio logístico.

\section{Referências}

BONNEVILLE-HÉBERT, A.; BOUCHARD, E.; TREMBLAY, D. D.; LEFEBVRE, R. Effect of reproductive disorders and parity on repeat breeder status and culling of dairy cows in Quebec. The Canadian Journal of Veterinary Research, Ottawa, v. 75, n. 2, p. 147-151, jun. 2011.

CORASSIN, C. H.; MACHADO, P. F.; COLDEBELLA, A.; SORIANO, S. Fatores de risco associados a falhas de 
concepção ao primeiro serviço em vacas leiteiras de alta produção. Acta Scientiarum. Animal Sciences, Maringá, v. 31, n. 3, p. 311-317, 2009.

DEGUILlAUME, L.; GEFFRÉ, A.; DESQUILBERT, L.; DIZIEN, A.; THOUMIRE, S.; VORNIÉRE, C.; CONSTANT, F.; FOURNIER, R.; CHASTANTMAILLARD, S. Effect of endocervical inflammation on days to conception in dairy cows. Journal of Dairy Science, Champaign, v. 95, n. 4, p. 1776-83, abr. 2012.

DUBUC, J.; DUFFIELD, T.F.; LESLIE, K. E.; WALTON, J. S.; LEBLANC, S. J. Definitions and diagnosis of postpartum endometritis in dairy cows. Journal of Dairy Science, Champaign, v. 93, n. 11, p. 5225-5233, jul. 2010.

FERGUSON, J. D.; GALLIGAN, D. T.; THOMSEN, N. Principal descriptors of body condition in Holstein dairy cattle. Journal of Dairy Science, Champaign, v. 77, n. 9, p. 2695-2703, 1994.

FERREIRA, A. M. Diagnóstico reprodutivo em bovinos, associando exame ginecológico e condição corporal. In: Reprodução da fêmea bovina - fisiologia aplicada e problemas mais comuns: causas e tratamento. Valença: Editora Editar, 2010. p. 129-163.

Efeito da amamentação e do nível nutricional na atividade ovariana de vacas mestiças leiteiras. 1990. Tese (Doutorado em Zootecnia) - Universidade Federal de Viçosa, Viçosa.

GAUTAM, G.; NAKAO, T. Prevalence of urovagina and its effects on reproductive performance in Holstein cows. Theriogenology, Stoneham, v. 71, n. 9, p. 1451-1461, 2009.

GILBERT, R. O.; SHIN, S. T.; GUARD, C. L.; ERB, H. N.; FRAJBLAT, M. Prevalence of endometritis and its effects on reproductive performance of dairy cows. Journal of the American Veterinary Medical Association, Schaumburg, v. 64, n. 9, p. 1879-1888, 2005.

GILBERT, R. O.; WILSON, D. G.; LEVINE, S. A.; BOSU, W. T. Surgical management of urovagina and associated infertility in a cow. Journal of the American Veterinary Medical Association, Schaumburg, v. 194, n. 7, p. 931-2, 1989.

GROOMS, D. L. Programas para controle de doenças infecciosas e melhoria do desempenho reprodutivo. In: CURSO NOVOS ENFOQUES NA PRODUÇÃO E REPRODUÇÃO DE BOVINOS, 14., 2010. Uberlândia. Anais... Uberlândia: Conapec Jr - UNESP, 2010. p. 418427. Disponível em: <http://www.conapecjr.com.br/ index.php>. Acesso em :11 fev. 2013.

GUSTAFSSON, H.; EMANUELSON, U. Characterisation of the repeat breeding syndrome in swedish dairy cattle. Acta Veterinaria Scandinavica, Copenhagen, v. 43, n. 2, p. 115-125, jun. 2002.

KAUFMANN, T. B.; DRILLICH, M.; TENHAGEN, B. A.; FORDERUNG, D.; HEUWIESER, W. Prevalence of bovine subclinical endometritis $4 \mathrm{~h}$ after insemination and its effects on first service conception rate. Theriogenology, Stoneham, v. 71, n. 2, p. 385-391, jan. 2009.

OPSOMER, G.; GROHN, Y. T.; HERTL, J.; CORYN, M.; DELUYKER, H.; DE KRUIF, A. Risk factors for post partum ovarian dysfunction in high producing dairy cows in Belgium: a field study. Theriogenology, Stoneham, v. 53, n. 4, p. 841-857, mar. 2000.

PAISLEY, L. G.; MICKELSEN, W. D.; ANDERSON, P. B. Mechanisms and therapy for retained fetal membranes and uterine infections of cows: a review. Theriogenology, Stoneham, v. 25, n. 3, p. 353-381, mar. 1986.

PRYCE, J. E.; COFFEY, M. P.; BROTHERSTONE, $\mathrm{S}$. The genetic relationship between calving interval, body condition score and linear type and management traits in registered Holsteins. Journal of Dairy Science, Champaign, v. 83, n. 11, p. 2664-2671, nov. 2000.

SALASEL, B.; MOKHTARI,A.; TAKTAZ, T.Prevalence, risk factors for and impact of subclinical endometritis in repeat breeder dairy cows. Theriogenology, Stoneham, v. 74, n. 7, p. 1271-1278, oct. 2010.

SANTOS, T. M. A.; CAIXETA, L. S.; MACHADO, V. S.; RAUF, A. K.; GILBERT, R. O.; BICALHO, R. C. Antimicrobial resistance and presence of virulence factor genes in Arcanobacterium pyogenes isolated from the uterus of postpartum dairy cows. Veterinary Microbiology, Amsterdam, v. 145, n. 1-2, p. 84-89, 2010.

SENOSY, W.; UCHIZA, M.; TAMEOKA, N.; IZAIKE, Y.; OSAWA, T. Evaluation of reproductive tract infection during early post-partum period and its relationship with subsequent reproductive performance in high milk producing dairy cows. Reproduction in Domestic Animals, Berlin, v. 47, n. 2, p. 203-207, abr. 2012.

SHELDON, I. M.; DOBSON, H. Postpartum uterine health in cattle. Animal Reproduction Science, Amsterdam, v. 82-83, n. 1, p. 295-306, jul. 2004.

SHELDON, I. M.; WILLIAMS, E. J.; MILLER, A. N. A.; NASH, D. M.; HERATH, S. Uterine diseases in cattle after parturition. The Veterinary Journal, London, v. 176, n. 1, p. 115-121, abr. 2008.

VASCONCELOS, J. L. M.; ESPER, C. R.; SANTOS, R. M.; VASCONCELOS, C. G. C.; WECHSLER, F. S. Detecção de subfertilidade em vacas leiteiras por meio de medidas anatômicas da região pélvica e do aparelho 
genital. Arquivo Brasileiro de Medicina Veterinária e Zootecnia, Belo Horizonte, v. 52, n. 5. p. 468-474, out. 2000.

VIEIRA, S. Bioestatística - tópicos avançados. 2. ed. Rio de Janeiro: Elsevier, 2003. 225 p.

YUSUF, M.; NAKAO, T.; RANASINGHE, R. M. S. B. K.; GAUTAM, G.; LONG, S.T.; YOSHIDA, C.; KOIKE,
K.; HAYASHI, A. Reproductive performance of repeat breeders in dairy herds. Theriogenology, Stoneham, v. 73, n. 9, p. 1220-1229, jan. 2010.

YUSUF, M.; RAHIM, L.; ASJA, M. A.; WAHYUDI, A. The Incidence of repeat breeding in dairy cows under tropical condition. Journal of Animal Science and Technology, Polones, v. 35, n. 1, p. 28-31, abr. 2012. 